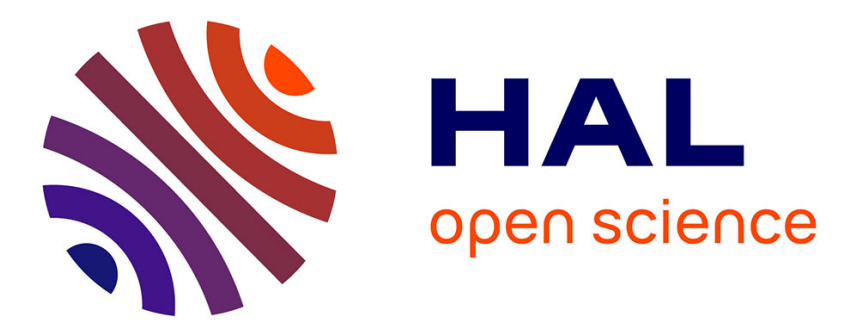

\title{
Nitrogen impurities and fluorescent NVcenters in detonation nanodiamonds: identification and distinct features
}

V. Yu. Osipov, S. Abbasi Zargaleh, François Treussart, K. Takai, N. Romanov, F. Shakhov, A. Baldycheva, Osipov V Yu, Zargaleh S Abbasi

\section{To cite this version:}

V. Yu. Osipov, S. Abbasi Zargaleh, François Treussart, K. Takai, N. Romanov, et al.. Nitrogen impurities and fluorescent NVcenters in detonation nanodiamonds: identification and distinct features. Journal of Optical Technology, 2019, 86 (1), pp.1. 10.1364/jot.86.000001 . hal-03142728

\section{HAL Id: hal-03142728 \\ https://hal.science/hal-03142728}

Submitted on 16 Feb 2021

HAL is a multi-disciplinary open access archive for the deposit and dissemination of scientific research documents, whether they are published or not. The documents may come from teaching and research institutions in France or abroad, or from public or private research centers.
L'archive ouverte pluridisciplinaire HAL, est destinée au dépôt et à la diffusion de documents scientifiques de niveau recherche, publiés ou non, émanant des établissements d'enseignement et de recherche français ou étrangers, des laboratoires publics ou privés. 
Nitrogen impurities and fluorescent $\mathrm{NV}^{-}$centers in detonation nanodiamonds: identification and distinct features

\title{
Osipov V.Yu. ${ }^{1,2 *}$, Abbasi Zargaleh S. ${ }^{3}$, Treussart F. ${ }^{3}$, TaKai K. ${ }^{2}$, Romanov N.M. ${ }^{4}$, Shakhov F.M. ${ }^{1}$, Baldycheva A. ${ }^{5}$
}

\author{
${ }^{1}$ Ioffe Institute, Polytechnicheskaya 26, St.Petersburg, 194021, Russia \\ ${ }^{2}$ Department of Chemical Science and Technology, Hosei University, 3-7-2, Kajino, Koganei, \\ Tokyo, 184-8584, Japan \\ ${ }^{3}$ Laboratoire Aimé Cotton, CNRS, Université Paris-Sud, ENS Paris-Saclay, Université Paris- \\ Saclay, 91405 Orsay, France \\ ${ }^{4}$ Peter the Great Polytechnical University, Polytechnicheskaya 29, St.Petersburg 195251, \\ Russia \\ ${ }^{5}$ College of Engineering Mathematics and Physical Sciences, University of Exeter, Exeter, \\ EX4 $4 Q F, U K$ \\ * E-mail: osipov@mail.ioffe.ru
}

\begin{abstract}
We show that nitrogen is the main impurity contained in detonation nano-diamonds (DNDs), at a concentration of $\sim 16000 \mathrm{ppm}$. The content of nitrogen-vacancy $\left(\mathrm{NV}^{-}\right)$centers in these DNDs is $\sim 2.7 \mathrm{ppm}$, which is the largest of all known types of nano-diamonds of size $<10 \mathrm{~nm}$ with artificially created $\mathrm{NV}^{-}$centers. The removal of graphite-like fragments from the DND surface allowed us to detect the characteristic photoluminescence (PL) of the $\mathrm{NV}^{-}$color centers in individual DND aggregates of sizes from 50-100 to 500-700 nm. We have further confirmed the detection of the negatively charged $\mathrm{NV}^{-}$through the observation of a strong decrease in the PL intensity when an external magnetic field is applied. Such an effect results from the optically detectable magnetic resonance of the electronic spin triplet ground state of $\mathrm{NV}^{-}$, that cannot be observed in other emitting defects, in a similar spectral range, including the neutral $\mathrm{NV}^{0}$ centers.
\end{abstract}

Codes OCIS: (160.4236) Nanomaterials; (160.2540) Fluorescent and luminescent materials; (300.6280) Spectroscopy, fluorescence and luminescence; (300.6370) Spectroscopy, microwave; 240.6675 (Surface photoemission and photoelectron spectroscopy), 280.1545 (Chemical analysis), (350.3850) Materials processing

\footnotetext{
*corresponding author, E-mail: osipov@mail.ioffe.ru
} 


\section{Introduction}

Fluorescent micro- and nanodiamonds (NDs) containing $\mathrm{NV}^{-}$centers are novel objects that have a number of promising innovative applications in magnetic sensing [1,2], cellular imaging and sensing [3], optical telecommunications [4], and quantum information processing [5]. This wide range of applications arises from the unique properties of the negatively charged $\mathrm{NV}^{-}$color centers with triplet spin properties that can be detected optically (Optically Detectable Magnetic Resonance, ODMR) [6] and great potential use of nanodiamonds (size <40 nm) in advanced biomedical applications [7-9]. The use of $\mathrm{NV}^{-}-$ containing ultra-small detonation nanodiamonds (DND, size $<6 \mathrm{~nm}$ ) in magnetic sensing applications at the nanoscale is still questioned due to a high concentration of various defects and imperfections in such nanocrystals [10]. Indeed, DNDs are formed during the process of detonation of a mixture of trinitrotoluene and hexogen under conditions of negative oxygen balance [11]. Synthesis is carried out in a very short time- on the order of microseconds. During this time, diamond crystallites up to $5 \mathrm{~nm}$ in size, with characteristically high levels of defects, are self-assembled from the decomposition products of the explosive mixture components- i.e. predominantly carbon. The details of the synthesis have been extensively described in the literature [11]. Nitrogen, which is one of the constituent elements of the components of the explosive mixture, also incorporates into the diamond lattice during the synthesis. It readily diffuses within the lattice structure, replacing carbon atoms, resulting in the existence of unpaired antibonding electron orbitals, which in turn gives rise to the $S=1 / 2$ paramagnetism of the nitrogen-doped diamond crystal. In principle, nitrogen impurities can reside in a diamond lattice in a variety of forms - an isolated neutral state ( $\mathrm{C}$ or P1 centers), a dimerized form (pairs of nitrogen atoms located at adjacent lattice sites: A centers) and numerous nitrogen-vacancy clusters, consisting of different numbers of nitrogen atoms and vacancies. The most important clusters, known for most types of synthetic and natural diamond crystals, include NV, NVN and 4NV centers. The latter, for example, represents four substitutional nitrogen atoms in a tetrahedral environment around a central vacancy. NV and NVN centers cause bright and stable fluorescence of diamonds in the red and green regions of the spectrum respectively. These centers can be purposefully induced in diamond crystals with nitrogen doping concentrations up to $~ 200$ ppm by irradiation with high-energy (2-15 MeV) electrons, creating vacancies in the diamond lattice, and by the subsequent annealing- at temperatures $\approx 800-950{ }^{\circ} \mathrm{C}$, which makes the vacancies mobile and allow their capture by substitutional nitrogen atoms [12]. As a result, NV and NVN centers appear in the 
diamond lattice. In detonation diamonds, vacancies are naturally present as results of stacking faults and inaccuracies that occur during the rapid assembly of a diamond lattice from $\mathrm{CH}_{3}{ }^{*}$ radicals, which are detonation products after pyrolysis. Therefore, in addition to nitrogen impurities, DND particles also contain isolated vacancies, multivacancies and NV type defect centers [10].

Multivacancies and $\mathrm{NV}^{-}$centers were successfully identified in DNDs by the method of electron paramagnetic resonance (EPR) in recent works of the authors [13-15]. Although certain EPR signals in DNDs were recorded back in 2002-2003, at the beginning of extensive research of this material, it took almost 10 years to understand and correctly assign them via exclusion of all possible parasitic factors associated with the impurity background and other types of incurred defects [13]. Moreover, studies combining different analysis methods, including: EPR spectroscopy, Raman spectroscopy, confocal fluorescence spectroscopy and X-ray photoelectron spectroscopy (XPS), have not been focused to date on the topic of NV centers within DNDs. In this work, we report results on the first complete investigations of DND particles that demonstrate specific photoluminescence (PL) of $\mathrm{NV}^{-}$ centers in DND, and also their sensitivity to an external magnetic field, related to their unique ODMR property.

\section{Experimental}

\subsection{Fabrication Methods}

DND powders were obtained by standard technology which includes blasting of an explosive mixture of trinitrotoluene-hexogen under conditions of negative oxygen balance. Synthesis and primary purification of the product was carried out by the commercial manufacturer the Federal State Unitary Enterprise "SCTB" Technolog " and the Ioffe Physical-Technical Institute, St. Petersburg, Russia. The unique technological process for powder synthesis offered by this manufacturer, made it possible to obtain diamond nanoparticles with a coherent scattering area size slightly exceeding $5 \mathrm{~nm}$. The X-ray powder diffraction (XRD) study demonstrated a coherent scattering size of selected sample of synthesized diamond nanoparticles up to $5.6 \mathrm{~nm}$.

The DND powders were further purified in acid mixtures to remove residual impurities of $3 d$ ferromagnetic metals for more accurate EPR and XPS studies. An additional surface cleaning by annealing of the DND powders in air at $430^{\circ} \mathrm{C}$ for $10-12$ hours was required for Raman scattering and subsequent photoluminescence studies [16]. For Raman 
studies samples were prepared by "pressing powder" into a cylinder of $3 \mathrm{~mm}$ in diameter and of no more than $2 \mathrm{~mm}$ thick inside a stainless steel cage.

Samples for confocal fluorescence microscopy studies were fabricated from the DNDs aqueous suspensions, that were readily obtained by dispersing the annealed powder in water at a concentration of $\approx 1 \mathrm{mg} / \mathrm{mL}$. The DNDs suspensions were diluted in water by a factor of 100 and spincoated onto a $170 \mu \mathrm{m}$ thick glass coverglass (Nexterion, Schott, Germany), which was precleaned in a mixture of ethanol and acetone in ultrasonic bath. Finally, for an accurate photoluminescence study, DND aggregates deposited on coverglass substrates were cleaned with a UV/O-Cleaner (low pressure mercury vapor grid lamp: 28,000 $\mu \mathrm{W} / \mathrm{cm}^{2}$ at $254 \mathrm{~nm}$ wavelength; model 42-220 from Jelight, USA) for 15 minutes. Under UV light exposure the process of efficient oxidation of graphite-like $s p^{2}$ phase around the DNDs with oxygen and/or ozone occurs, which is followed by gas-phase etching of the surface with substantial removal of $s p^{2}$ carbon. The nanostructured $s p^{2}$-carbon absorbs optical radiation and, therefore, is extremely undesirable for PL investigation from DND particles. Exposure of the substrate with deposited DND particles to UV radiations also leads to the removal of the parasitic organic compounds, which can create some luminescent background.

\subsection{Characterization Methods}

\subsubsection{Electron paramagnetic resonance (EPR)}

The EPR spectra of the samples were recorded at room temperature within the microwave X-band at a frequency of $\sim 9.5 \mathrm{GHz}$ using EPR spectrometer JEOL JES-FA 300 (Japan). Powder in the amount of 50-60 mg was introduced into a EPR quartz tube of $4 \mathrm{~mm}$ in diameter. The height of the powder column in the tube did not exceed $10 \mathrm{~mm}$. The open end of the tube was sealed against moisture.

The EPR spectra with $g$-factors in the range 4.00-4.30 were recorded with microwave power of $P_{\mathrm{Mw}}=10 \mathrm{~mW}$, magnetic field modulation amplitude $A_{\mathrm{m}}=10 \mathrm{G}$ and frequency $v=100 \mathrm{kHz}$, the gain, $G \sim 10^{3}$, and the signal accumulation $N=16$. These parameters were chosen to obtain the optimal signal-to-noise ratio. The time constant was $0.030 \mathrm{~s}$, the total recording time for the magnetic field sweep in interval from 1300 to $2000 \mathrm{G}$ was 120 seconds. At microwave power $P_{\mathrm{MW}}<50 \mathrm{~mW}$, the intensity of the EPR signal with the $g$-factor $g \approx 4$ followed a linear dependence of $I_{\mathrm{pp}} \sim\left(P_{\mathrm{MW}}\right)^{1 / 2}$ and there was no signal distortion from saturation. The concentration of paramagnetic $\mathrm{NV}^{-}$centers was estimated by double integration of the corresponding EPR signal with $g=4.26$, followed by assignment to 
the corresponding sample weight and comparison to the reference EPR signal(s) from reference sample(s). We chose one reference DND sample with well-defined integrated intensity of the $g=2.0027 \mathrm{EPR}$ line and with previously independently determined $S=1 / 2$ paramagnetic center's concentration of $6.3 \times 10^{19}$ centers/g $(1260 \mathrm{ppm})$ by precision measurements of the magnetization field dependence at low temperatures using a SQUID ${ }^{1}$ magnetometer. In addition, a synthetic fluorescent Ib HPHT diamond powder with calculated integral intensity of the $g=4.26 \mathrm{EPR}$ line and known concentration of $S=1 \mathrm{NV}^{-}$centers of $5 \mathrm{ppm}$ was also used as an independent reference for correct determination of the obtained concentration of $\mathrm{NV}^{-}$in DND [17]. The EPR $g=4.26$ line was recorded for this reference sample with the following parameters: $P_{\mathrm{MW}}=1 \mu \mathrm{W}, A_{\mathrm{m}}=1.6 \mathrm{G}, N=25$ in a mode far from saturation.

\subsubsection{X-ray photoelectron spectroscopy (XPS)}

X-ray photoelectron spectroscopy (XPS) of DNDs was performed using PerkinElmer PHI 5600 Multi-Technique system, equipped with a source of monochromatic $\mathrm{Al} \mathrm{K \alpha}$ radiation (photon energy $1486.6 \mathrm{eV}$ ) and a hemispherical energy analyzer. The angle of incidence of X-rays on the sample was $45^{\circ}$. The spectral characteristics ${ }^{2}$ of photoelectrons were obtained after analyzing of photoelectrons flux emitted with different kinetic energies from a sample area of $\sim 1 \mathrm{~mm}^{2}$ [18]. Detected photoelectrons are emitted by the material surface- a few atomic layers thick top layer (no more than 5-10 nm). Particular attention was paid to analyses of the areas of photoemission peaks of carbon $\mathrm{C} 1 \mathrm{~s}$, nitrogen $\mathrm{N} 1 \mathrm{~s}$ and oxygen O1s. Obtained spectral characteristics were processed in MultiPak v8.2. Decomposition of photoemission peaks into individual components (contours) was performed after subtraction of the background followed by approximation of the shape of the peaks by a set of Gaussian or mixed-type singlet contours. Spectra of the DND sample were taken before and after etching of the surface with argon ions. Ion etching was carried out for 3 minutes at an accelerating voltage of $3 \mathrm{kV}$ and an ion beam current of about $4 \mu \mathrm{A}$. The Ar ion etching was used not only for cleaning of the sample surface from adsorbed gases and other chemisorbed atomic complexes, but also to study composition and distribution of elements covalently bonded to diamond surface. Charging effect during measurements was eliminated by electron beam irradiation of the samples.

\footnotetext{
${ }^{1}$ Superconducting quantum interference device

2 The number of detected electrons emitted in a single energy interval, depending on their kinetic energy.
} 


\subsubsection{Raman scattering}

Spectra of secondary radiation, i.e. luminescent radiation together with Raman scattering, were collected using $532 \mathrm{~nm}$ (power $0.5 \mathrm{~mW}$ ) and $325 \mathrm{~nm}^{3}$ (power $0.8 \mathrm{~mW}$ ) excitation laser wavelengths of a home-built micro-Raman system. Secondary radiation was collected in the backscattering geometry with laser spot of diameter $\sim 2 \mu \mathrm{m}$ focused on the surface of sample using 50× microobjective for $532 \mathrm{~nm}$ excitation wavelength and using specially designed UV microscope lens with 30-36 accumulations for $325 \mathrm{~nm}$ excitation wavelength. In studies using both excitation lasers, diffraction gratings with grooves densities of $600 \mathrm{~mm}^{-1}$ (532 nm laser) and $1800 \mathrm{~mm}^{-1}$ (325 nm laser) were used.

\subsubsection{Confocal fluorescence microscopy}

The PL setup used to map PL from DND particles spincoated on coverglass consists of a home-made inverted confocal scanning microscope combined with an imaging spectrograph. The confocal microscope includes a piezoelectric scanning stage (NanoPDQ75, Mad City Labs Inc., USA), a high-numerical aperture microscope objective (PlanApo 100×, $\mathrm{NA}=1.40$, Nikon, Japan), an optically pumped semiconductor laser (Verdi, Coherent Inc., USA) emitting at $532 \mathrm{~nm}$ wavelength that is injected into the objective through a long-pass interferential dichroic mirror (z532rdc, Semrock, USA). After a $50 \mu \mathrm{m}$ diameter confocal pinhole, a bandpass filter (FF01-697/75, Semrock) is inserted on the detection path to reject the remaining excitation light. A single photon counting module (SPCM-AQR-14, PerkinElmer, Canada) is used as a point detector. The excitation radiation from laser is circularly polarized and has the power of $0.5 \mathrm{~mW}$. The imaging spectrograph is composed of a concave grating imaging the spectrum on a back-illuminated cooled CCD silicon array detector (DU440-BU2, Andor Technologies, UK), leading to a spectral resolution of about $1 \mathrm{~nm}$.

Mapping of the luminescence signal was carried out in a square of $20 \times 20 \mu \mathrm{m}^{2}$. To remove the PL spectrum in the range of 500-900 nm, one of several DND aggregates deposited on the substrate (within the light spot of the microobjective of $100 \times$ ) with transverse dimensions of only 0.6 microns and having intense luminescence was selected. The exposure time was $60 \mathrm{~s}$ including the removal of one spectrum. The magnetic field was

\footnotetext{
${ }^{3}$ Excitation wavelength of $325 \mathrm{~nm}$ was used only for Raman spectroscopy measurements of detonation diamond. In this case, the contribution to Raman scattering signal from collective defects of the graphite-like $s p^{2}$ phase is significantly reduced in the region $1350-1400 \mathrm{~cm}^{-1}$ (so-called $D$ band), and the sample luminescence is also suppressed comparing with the case of $532 \mathrm{~nm}$ excitation.
} 
applied using an iron-neodymium-bore doped permanent magnet brought at a few millimeters distance to the coverglass sample. Its magnitude at the sample location was about $\sim 900 \mathrm{G}$. The time dependence of the luminescence signal was recorded with repeated exposure and removal of the magnet.

\section{Experimental results and discussion}

\subsection{Identification of $\mathrm{NV}^{-}$centers in DND and measurement of their concentration}

Since the radiation emitted by centers can be absorbed by neighboring absorbing agents and phases surrounding the particles (e.g. graphitic coverage and metal inclusions), it is very important to control and monitor $\mathrm{NV}^{-}$centers in diamonds by non-optical methods independently. The negatively charged $\mathrm{NV}^{-}$centers can be detected by EPR spectroscopy even in case of absence their photoluminescence and their total concentration can be easy evaluated by analysis the EPR spectrum $[14,19,20]$. At the same time, the evaluated total $\mathrm{NV}^{-}$ centers concentration provides valuable information on the potential brightness of $\mathrm{NV}^{-} \mathrm{PL}$ after extensive purification. The EPR spectrum of the DND powder purified from $3 d$ transition metal magnetic impurities (mostly $\mathrm{Fe}, \mathrm{Ni}$ ) in the region of the so-called half magnetic field corresponding to $g$-factor $g \sim 4$ is shown in Fig 1. The spectrum consists of two close lines with $g$-factors $g_{1}=4.26$ and $g_{2}=4.00$, and widths $\Delta H_{\mathrm{pp} 1}=28 \mathrm{G}$ and $\Delta H_{\mathrm{pp} 2}=$ 13.5 G. The distance between the lines is about $100 \mathrm{G}$. The first low field line, corresponds to the forbidden transitions $\Delta M_{s}=2$ between the Zeeman energy levels of the triplet state of the $\mathrm{NV}^{-}$center in a magnetic field, and the second, high-field, to the same forbidden transitions $\Delta M_{s}=2$, which occurs when microwave radiation is absorbed by multivacancies. In ref. 14 these lines were assigned to triplet centers in the DNDs, and in particular the $g_{1}=4.26$ line was assigned to $\mathrm{NV}^{-}$center. The position of this $g_{1}=4.26$ line is highly sensitive to the microwave frequency, and can be shifted by $\sim 0.5 \%$ upon a small frequency change from 9.0 to $9.9 \mathrm{GHz}$ (X-band). This sensitivity is due to the large value of the zero-field splitting parameter $D\left(D=850 \times 10^{-4} \mathrm{~cm}^{-1}\right)$, which is related to the exchange interaction of constituting spins in the spin Hamiltonian of the triplet center [19]. The position of the second line $\left(g_{2}=4.00\right)$ is practically independent to frequency changes, and the parameter $D$ of the corresponding triplet center (multivacancy) is approximately 5-6 times smaller. The integral intensity of the line $g_{1}=4.26$ can be used to precisely estimate the concentration of $\mathrm{NV}^{-}$ centers even when the luminescence of nanodiamonds is not detectable due to absorption by graphite-like atomic shells. Using a synthetic submicron diamond with a known 
concentration of $\mathrm{NV}^{-}$centers as a reference sample, the concentration of $\mathrm{NV}^{-}$centers in the DNDs was determined to be $2.7 \mathrm{ppm}$. Additionally, we found that main technological treatments at temperatures below $500{ }^{\circ} \mathrm{C}$ (in vacuum, in air and even in fluorine gas) have no effect on the concentration of $\mathrm{NV}^{-}$centers in the DNDs. This is because the EPR spectrum of DND in the half-magnetic field region is practically not changed and has almost the same double integrated intensity of signal $g_{1}=4.26$ after described treatments. This indicates that the $\mathrm{NV}^{-}$centers are buried from the nanoparticle surface at least by the lattice constant $(\sim 0.36 \mathrm{~nm})$ and are not in reaction with most of external chemical agents that do not etch the diamond phase. Only high temperature treatment at $900-1000^{\circ} \mathrm{C}$ leads to disappearance of the $\mathrm{NV}^{-}$centers due to their significant modification or unification into larger aggregates and complexes due to the diffusion of vacancies.

The main EPR signal of the DND has a Lorenzian curve profile of linewidth $\Delta H_{\mathrm{pp}}=8.4 \mathrm{G}$ [13] wider than the one of fluorescent nanodiamonds (FNDs) milled from microcrystalline diamond synthesized by high pressure and high temperature (HPHT) method. This broad signal can be explained by more larger exchange and dipole-dipole interactions between the spins $S=1 / 2$ in spin ensemble within individual DND nanoparticle than within FND. The dipole-dipole interaction between spins is inversely proportional to the third power of the distance between them and does not depend on the medium (vacuum or solid). The intensity of the main EPR signal collected from all paramagnetic centers $S=1 / 2$, both of nitrogen (P1 centers [21]) and non-nitrogen origin, indicates a spin concentration of $\sim 1300$ ppm, corresponding ${ }^{1}$ to $\sim 15$ of $S=1 / 2$ spins in each DND [13]. The exchange interaction neutralizes the individual features of different interior spins $1 / 2$, erases the difference between them and the consolidated Lorentzian ESR signal of $g=2.0027$ indicates paramagnetic centers of pseudo-single origin. However, it can be concluded from obtained data that approximately $40-50 \%$ of the total number of all paramagnetic centers in the DNDs is due to unpaired antibonding orbitals of isolated nitrogen dopants (P1 centers). The experimental concentration estimate of $\mathrm{NV}^{-}$centers in the DNDs is approximately $~ 500$ times smaller than the concentration of all $S=1 / 2$ paramagnetic defects in the system, and approximately 220 times smaller than the estimated concentration of isolated nitrogen atoms.

This concentration ratio for isolated paramagnetic nitrogen $(\mathrm{P} 1)$ and $\mathrm{NV}^{-}$centres is qualitatively consistent with the process of NV center formation: from "randomly built-in"

\footnotetext{
${ }^{1}$ We assume that each DND consists of $\sim 11,000$ carbon atoms.
} 
during the $\sim 0.5 \mu$ s duration of the synthesis to the growing diamond nanoparticle volume both substitutional nitrogen atoms and vacancies. Here, we assumed that each $\mathrm{NV}^{-}$center is formed as a result of random occurrence of substitutional nitrogen atoms and vacancies in the nearest neighbour lattice sites during detonation synthesis and subsequent rapid cooling to temperatures of the order of $\sim 500{ }^{\circ} \mathrm{C}$. Thus, the diffusion range of vacancies in the lattice during such short synthesis does not exceed few nanometers.

\subsection{Determination of Nitrogen Impurities Concentration}

The complete understanding of the DNDs composition (both surface and bulk) requires the knowledge of concentration of all impurities, which can be precisely determined using X-ray photoelectron spectroscopy (XPS). XPS is a powerful tool for studying the chemical state of main alien elements in bulk and on the surface of the DNDs [22]. The XPS signals of carbon $(\mathrm{C} 1 \mathrm{~s})$, oxygen $(\mathrm{O} 1 \mathrm{~s})$ and nitrogen $(\mathrm{N} 1 \mathrm{~s})$ have been recorded before and after etching of the surface with Ar ions. The data indicate the presence of oxygen-containing atomic groups - hydroxyl, carboxyl and carbonyl groups at DND surface. The concentration of surface oxygen and inner nitrogen was determined to be $\sim 5.5$ at $\%$ and $\sim 1.6$ at $\%$ respectively. In addition to these elements, photoemission signals from Silicon (Si2p), Titanium (Ti2p), Aluminium (A12p) were also recorded, but the concentration of the first two did not exceed 0.1 at. \%, and the aluminium concentration was 0.21 at.\% [23]. However, our main interest was focused on the XPS signal from nitrogen, since nitrogen is a main inner impurity. The photoemission peak of nitrogen (N1s) before and after etching by Ar ions is shown in Fig.2. Etching with Ar ions (accelerated to $3 \mathrm{keV}$ energy) for 3 minutes results in the removal of weakly bound chemical sorbents and physically adsorbed compounds from the surface, while the diamond covalent lattice remains not affected. The characteristic highenergy peaks $(404.9 \mathrm{eV}$ and $407.3 \mathrm{eV})$ strongly decrease or even disappear in N1s photoemission signal after Ar ion etching, which demonstrates presence of nitrate-ions and nitrite-groups on the untreated surface. At the same time, the strong decrease of the N1s peak at $\sim 407 \mathrm{eV}$ in the XPS spectral characteristics indicates complete or partial removal of nitrate-ions from the surface (Fig.2b). In fact, nitrate-ions are chemical sorbents bound to the surface through defective sites. They are formed at the stage of synthesis and chemical purification of DNDs from graphite-like phase in nitric acid. In Fig. $2 b$ the peak at $\sim 405 \mathrm{eV}$ is suppressed only partially, which indicates that even after Ar ion etching there is a stronger bond between the corresponding nitrite groups and the surface. The main peak of N1s signal 
at $\sim 401 \mathrm{eV}$, remains after ion beam treatment and corresponds to chemical bonds of the type $\mathrm{N}-s p^{3}-\mathrm{C}$. This peak is a main characteristic of nitrogen impurity elements covalently bound to the diamond lattice. This peak signal appears due to all forms of nitrogen present in the diamond matrix, including NN nitrogen dimers, next nearest neighbouring or distant, farlying NN pairs, and more complex clusters of few nitrogen atoms. It is worth to note, that the photoemission peak $\mathrm{N} 1 \mathrm{~s}$ in the low-energy region at $\sim 398-399 \mathrm{eV}$, which usually corresponds to the covalent bonds of nitrogen with carbon in the $s p^{2}$ hybridization of $\mathrm{N}-s p^{2}-$ $\mathrm{C}$, is not present in the XPS spectrum. This indicates both a very small amount of carbon in the $s p^{2}$ coordination on the surface of DNDs, and a significant localization of nitrogen in $s p^{3}$ crystalline lattice within diamond nanoparticles. Similar data were obtained for the photoemission peak of carbon C1s (see Ref. 23 for details). After ion etching, this signal consists of two main peaks: the one centred on $284.9 \mathrm{eV}$ corresponds to $\mathrm{C}-\mathrm{C}$ bonds in diamond matrix, and the peak centred on $287.3 \mathrm{eV}$ to chemical bonds $\mathrm{C}-\mathrm{N}$. The $\mathrm{C} 1 \mathrm{~s}$ peak at $288.6 \mathrm{eV}$, which corresponds to carbon in the carboxylic groups, disappears after ion etching: only diamond carbon and $s p^{3}$ carbon bound to nitrogen remains in the $\mathrm{C} 1 \mathrm{~s}$ signal. Our complex XPS analysis of $\mathrm{N} 1 \mathrm{~s}$ and $\mathrm{C} 1 \mathrm{~s}$ signals (together with $\mathrm{O} 1 \mathrm{~s}, \mathrm{Al} 2 \mathrm{p}$ and residual elements $(<0.1$ at.\% $)$ signals $)$ suggests that nitrogen is contained within the diamond lattice in an amount up to 1.6 at\% ( $16000 \mathrm{ppm})$ and mainly in the form of complex clusters which are not paramagnetic like dimers NN (A-centers) and therefore not EPR observable. A small part of these clusters represents isolated paramagnetic nitrogen atoms with spin $S=1 / 2$ and is accessible for observation by EPR. The total amount of paramagnetic nitrogen in the neutral state does not exceed $\sim 1300 \mathrm{ppm}$ according to the EPR data, i.e. $9 \%$ of all forms of nitrogen recorded in diamond lattice according to XPS data.

\subsection{Identification of diamond lattice from Raman spectrum}

Independent identification of crystalline diamond phase in DNDs was performed from Raman analysis extracted from the spectra of secondary radiation after subtracting the luminescent background. The DND PL spectrum recorded with excitation wavelength of $532 \mathrm{~nm}$ demonstrates a strong $\mathrm{NV}^{-}$center emission band in the interval 600-750 nm, and badly distinguishable characteristic Raman bands for diamond and graphite phases in the spectral interval $<600 \mathrm{~nm}$ (PL and Raman spectra obtained at $532 \mathrm{~nm}$ excitation are not presented here). The effective analysis of the weak Raman lines under $532 \mathrm{~nm}$ excitation wavelength is difficult due to strong luminescence background, while the subtraction of 
which reveals a large noise level in the Raman signal. The characteritic Raman peaks of DNDs (actual diamond peak at $\sim 1323 \mathrm{~cm}^{-1}$ with FWHM $=36 \mathrm{~cm}^{-1}$ and graphite $\mathrm{G}$ peak at $\sim 1590 \mathrm{~cm}^{-1}$ ) are obtained at resonant excitation of $325 \mathrm{~nm}$ for which the PL is reduced (Fig. 3). The Lorenz or Gaussian approximation applied to obtained experimental spectra allows to observe a band at $1299 \mathrm{~cm}^{-1}$ which overlaps the characteristic Raman band of $1323 \mathrm{~cm}^{-1}$ attributed to diamond lattice of finite length $(<5 \mathrm{~nm})$, and with a FWHM $\sim 220 \mathrm{~cm}^{-}$ ${ }^{1}$ much wider that the one of the $1323 \mathrm{~cm}^{-1}$ line. This $1299 \mathrm{~cm}^{-1}$ wide band can be attributed not only to amorphous carbon in a state of $s p^{2} / s p^{3}$ hybridization, but also to some fraction of fine diamond particles with a size of less than 2-3 nm [24,25]. Following Ref. 26 the highfrequency band at $\sim 1650 \mathrm{~cm}^{-1}$ can be attributed to Raman scattering by bending vibrations of surface O-H groups or even water molecules adsorbed on DND particles. All main Raman bands attributed to diamond lattice of few nanometer length, to graphite-like $s p^{2}$ phase, or to hydroxyl groups and physisorbed water at the sample surface are characterized by intensities of about the same order. However, the scattering cross-section of diamond and graphite phases differ by more than 50 times, which makes it impossible to quantitatively estimate the content of the graphite $s p^{2}$ phase in the material by this method. In accordance with other methods, e.g. nuclear magnetic resonance, the graphite phase content should not exceed $\sim 6-8$ at.\% [27]. This is achieved by exposure of the material to air at $430{ }^{\circ} \mathrm{C}$ for several hours. As a result, the gas-phase decomposition and removal of the $s p^{2}$ shell partially covering the DND surface occur. Removing $s p^{2}$ shells by gas-phase etching in air is a prerequisite for obtaining intense luminescence from $\mathrm{NV}^{-}$centers in DNDs. Thus, the presence of diamond Raman peak at $\sim 1323 \mathrm{~cm}^{-1}$ with FWHM $=36 \mathrm{~cm}^{-1}$ confirms the good crystalline quality of DND particles with size about $5 \mathrm{~nm}$.

\subsection{Confocal fluorescence microscopy and local probing sensitive to magnetic field.}

Registration of fluorescent radiation emitted by isolated DND particles or aggregates is crucial for understanding their potential use as fluorescent markers and nanosensors. Photoluminescence was recorded on DND aggregates spin-coated on a glass microscope coverslip. The average size of DND aggregates in the aqueous suspension before spin-coating was $30 \mathrm{~nm}$ (as measured by dynamic light scattering).

Figure 4a shows a PL 2D map obtained by confocal microscopy, using a $532 \mathrm{~nm}$ wavelength excitation laser. Bright spots corresponding to aggregates of DNDs particle up to $500-800 \mathrm{~nm}$ in size are observed, among dimmer spots of size limited by optical diffraction, corresponding to aggregates of smaller dimensions (in the range 50-100 nm). Obviously, the 
larger in size DND aggregates, contain higher number of luminescence centres ( $\mathrm{NV}^{-}$defects), because the PL intensity is approximately 7-8 times greater than the one of aggregates of smaller size. The PL spectrum of the aggregate surrounded by circle in Fig. 4a is shown in Fig. 4b. It indicates the presence of both $\mathrm{NV}^{-}$and $\mathrm{NV}^{0}$ defects, although their characteristic zero-phonon lines (ZPL) at $638 \mathrm{~nm}$ and $575 \mathrm{~nm}$ respectively are not detected. ZPLs are usually well resolved only for diamond crystals of micron size or larger, while for nanometerscale crystals it is usually not observed, due to quantization and subsequent substantial distortion of phonon modes. This effect is analogous to Raman line broadening upon transition from single crystals diamond to diamond nanoparticles of $<5 \mathrm{~nm}$ in size. To confirm unambiguously the presence of $\mathrm{NV}^{-}$centers and not only of $\mathrm{NV}^{0}$ in the DND, we studied the influence of an external magnetic field on the PL intensity. No PL modification is expected from $\mathrm{NV}^{0}$, which has no magneto-optical properties contrary to $\mathrm{NV}^{-}$. Figure 5 shows the time variation of the PL intensity from an isolated DND aggregate in the presence of an external magnetic field switch on by quickly approaching a compact permanent magnet from the DND ("ON", $\boldsymbol{B} \neq 0$ ) or by removing it ("OFF", $\boldsymbol{B}=0$ ). The PL time dependence has a square "telegraph-like" form due to the decrease of PL intensity when the magnetic field is applied (amplitude $\sim 900 \mathrm{G}$ ). This occurs as a result of mixing the $m s=0$ and $m s= \pm 1$ sublevels of the $\mathrm{NV}^{-}$centers and a corresponding change in their populations with magnetic field being "ON". More precisely, the $\mathrm{NV}^{-}$ground and excited states have a triplet symmetry, with the spin quantum number $m_{S}=0, \pm 1$ quantized along the $\mathrm{N}-\mathrm{V}$ axis in the crystal. The optical transitions between the ground and excited triplet states preserve the spin state $(\Delta m S$ $=0$ ). However, from the $m_{S}= \pm 1$ excited state, $\mathrm{NV}^{-}$also decays with no radiation in the visible domain, through a system of two singlet metastable states before coming back to the ground state [6]. This additional decay pathways results in a lower fluorescence intensity from transition within the $m_{S}= \pm 1$ subsystem of ground and excited triplet states, than within the $m_{s}=0$ subsystem, which is also at the origin of the ODMR signal. Incidentally, the same pathway is also responsible for the spin ground state polarization in the $m_{S}=0$ sub-state, under optical pumping at $532 \mathrm{~nm}$ wavelength. Moreover, in an external magnetic field $\boldsymbol{B}$, the energy degeneracy between $m_{S}= \pm 1$ levels is lifted and the ground and excited spin states $\left|m_{S}\right\rangle$ can be decomposed on the $|0\rangle$ and $| \pm 1\rangle$ basis. The coefficients of such linear combination are determined by the external magnetic field amplitude and direction and can be calculated from the $\mathrm{NV}^{-}$spin Hamiltonian in the $\boldsymbol{B}$ field. In the general case $\left|m_{S}\right\rangle$ is a mix of $\mid 0>$ and $\mid \pm 1>$ [28]. Therefore, compared to the situation of pure $\mid 0>$ state that results from 
optical pumping under $532 \mathrm{~nm}$ light excitation (and in absence of $\boldsymbol{B}$ ), a decrease of $\mathrm{NV}^{-}$ center fluorescence intensity is expected in the presence of an external magnetic field [29], as observed in Fig. 5.

\section{Conclusion}

In this article, we have shown that nitrogen is the main impurity in the DNDs. It is contained in the crystalline lattice of diamond in an amount of $\sim 16,000 \mathrm{ppm}$. The content of $\mathrm{NV}^{-}$centres is $\sim 2.7 \mathrm{ppm}$, which is the largest ever detected in this types of nano-diamonds of size $<10 \mathrm{~nm}$. The ultra-small dimensions cause $\mathrm{NV}^{-}$luminescence quenching and absorption of emitted radiation by various point and collective defects located near the surface. Annealing treatment of DNDs in air and/or in ozone allows to remove such defects associated to graphite-like fragments at DND surface and to detect the specific PL of $\mathrm{NV}^{-}$centres, both in DND powder and in individual DND aggregates. When an external magnetic field is switched "ON" and "OFF", an accompanying variation of the PL intensity is observed, which results from the sensitivity of the $\mathrm{NV}^{-}$triplet ground state to magnetic field and from the optically detectable magnetic resonance characteristic of this NV charge state only. The application of this effect to discriminate ultra-small DND (size $<10 \mathrm{~nm}$ ) in biological environment with large autofluorescence background (for enhancement the contrast of intercellular imaging) is promising [30]. Such DND could also be used as point optical probes of high resolution nanoscale magnetic field sensing and all-optical magnetic imaging [31]. 


\section{Figure captions.}

Fig.1. EPR spectra of acid-purified DNDs in the region of half magnetic field (blue curve) and of reference fluorescent Ib HPHT diamond nanoparticles up to $~ 100 \mathrm{~nm}$ in size (red curve). The low-field line with $g=4.26$ corresponds to $\mathrm{NV}^{-}$centers. Microwave frequency $9.4006 \mathrm{GHz}$.

Fig.2. XPS spectra of acid-purified DNDs: N1s photoemission peaks before (a) and after Ar ion etching (b).

Fig.3. Raman spectrum of DNDs after acid purification and air treatment at $430{ }^{\circ} \mathrm{C}$; wavenumbers of the Raman bands are shown. Excitation wavelength $325 \mathrm{~nm}$. The spectrum is decomposed into six components, where the bands at 1323 and $1590 \mathrm{~cm}^{-1}$ come from $\mathrm{sp}^{3}$ coordinated carbons of DND particles with mean size of $\sim 5 \mathrm{~nm}$ and $\mathrm{sp}^{2}$ phase, respectively, while the bands centered at $\sim 1745, \sim 1650$ and $\sim 1300 \mathrm{~cm}^{-1}$ are assigned to the contribution of surface carbonyl, hydroxyl groups and fraction of diamond particles with sizes less than 3 $\mathrm{nm}$, correspondingly. Weak shoulder at $\sim 1100 \mathrm{~cm}^{-1}$ comes from transpolyacetylene chains.

Fig.4. 2D- mapping of PL signal intensity of DND spin-coated on a glass microscope coverslip (a) and photoluminescence spectrum (b) of the selected aggregate of detonation nanodiamonds surrounded by a circle in (a). Laser excitation at $532 \mathrm{~nm}$ wavelength $(100 \mu \mathrm{W}$ excitation power). Integration time on the spectrometer CCD is $60 \mathrm{~s}$. The shape of this PL spectrum is consistent with the contribution of both $\mathrm{NV}^{-}$and $\mathrm{NV}^{0}$ photoluminescence although we cannot identify their characteristic zero-phonon lines.

Fig.5. Photoluminescence intensity versus time for the DND surrounded by a circle in Fig. 4 (scan), in the presence or absence of an external magnetic field. Excitation laser wavelength: $532 \mathrm{~nm}$, power: $100 \mu \mathrm{W}$. The laser is focused on the nanodiamond. In absence of external magnetic field $(B=0)$ we observe only small changes of intensity, that might be due to the blinking of some of the $\mathrm{NV}^{-}$color centers close to DND surface, and therefore more sensitive to charge state trapping. When we apply a magnetic field by manually approaching a permanent magnet at a distance from the sample at which the field amplitude is $\approx 900 \mathrm{G}$, we observe a large decrease of the PL intensity. 


\section{References}

[1] Balasubramanian $\mathrm{G}$ et al., "Nanoscale imaging magnetometry with diamond spins under ambient conditions," Nature 455, 648-651 (2008).

[2] J.M. Taylor, P. Cappellaro , L. Childress , L. Jiang, D. Budker, P.R. Hemmer, A. Yacoby, R. Walsworth and M.D. Lukin, "High-sensitivity diamond magnetometer with nanoscale resolution,” Nature Phys. 4, 810-816 (2008).

[3] Y. Y. Hui, L.-J. Su, O. Y. Chen, Y.-T. Chen, T.-M. Liu and H.-C. Chang, "Wide-field imaging and flow cytometric analysis of cancer cells in blood by fluorescent nanodiamond labeling and time gating," Sci. Rep. 4, 5574 (2014).

[4] C. Kurtsiefer, S. Mayer, P. Zarda and H. Weinfurter , "Stable solid-state source of single photons," Phys. Rev. Lett. 85, 290-293 (2000).

[5] M.V.G. Dutt, L. Childress, L. Jiang, E. Togan, J. Maze, F. Jelezko, A.S. Zibrov, P.R. Hemmer and M.D. Lukin, "Quantum register based on individual electronic and nuclear spin qubits in diamond," Science 316, 1312-1316 (2007).

[6] M. W. Doherty, N. B. Manson, P. Delaney, F. Jelezko, J. Wrachtrup, and L. C. L. Hollenberg, "The nitrogen-vacancy colour centre in diamond," Phys. Rep. 528 (1), 1-45 (2013).

[7] O. Shenderova and G. McGuire, "Science and engineering of nanodiamond particle surfaces for biological applications," Biointerphases 10, 030802 (2015).

[8] S. Nagarajan, C. Pioche-Durieu, L. H. G. Tizei, C.-Y. Fang, J.-R. Bertrand, E. Le Cam, H.-C. Chang, F. Treussart and M. Kociak, "Simultaneous cathodoluminescence and electron microscopy cytometry of cellular vesicles labeled with fluorescent nanodiamonds," Nanoscale 8, 11588 (2016).

[9] I. Rehor, J. Slegerova, J. Kucka, V. Proks, V. Petrakova, M.-P. Adam, F. Treussart, S. Turner, S. Bals, P. Sacha, M. Ledvina, A. M. Wen, N. F. Steinmetz, and P. Cigler, 
"Fluorescent nanodiamonds: fluorescent nanodiamonds embedded in biocompatible translucent shells," Small 10(6), 1106 (2014).

[10] B. R. Smith, D. W. Inglis, B. Sandnes, J. R. Rabeau, A.V. Zvyagin, D. Gruber, C. J. Noble, R. Vogel, E. Osawa, and T. Plakhotnik "Five-nanometer diamond with luminescent nitrogen-vacancy defect centers," Small 5 (14), 1649-1653 (2009).

[11] A. Vul' and O. Shenderova, eds., Detonation Nanodiamonds: Science and Applications (Pan Stanford, Singapore, 2014).

[12] L.-J. Su, C.-Y. Fang, Y.-T. Chang, K.-M. Chen, Y.-C. Yu, J.-H. Hsu and H.-C. Chang, "Creation of high density ensembles of nitrogen-vacancy centers in nitrogen-rich type Ib nanodiamonds," Nanotechnology 24, 315702 (2013).

[13] V. Yu. Osipov, A. I. Shames, T. Enoki, K. Takai, M. V. Baidakova, and A. Ya. Vul, "Paramagnetic defects and exchange coupled spins in pristine ultrananocrystalline diamonds," Diamond Relat. Mater. 16(12), 2035 (2007).

[14] A. I. Shames, V. Yu. Osipov, H. J. von Bardeleben, J.-P. Boudou, F. Treussart, and A. Ya. Vul', 'Native and induced triplet nitrogen-vacancy centers in nano- and microdiamonds: half-field electron paramagnetic resonance fingerprint," Appl. Phys. Lett. 104, 063107 (2014).

[15] V. Yu. Osipov, N. M. Romanov, K. V. Bogdanov, F. Treussart, C. Jentgens, and A. Rampersaud, "Investigation of $\mathrm{NV}^{(-)}$centers and crystallite interfaces in synthetic singlecrystal and polycrystalline nanodiamonds by optical fluorescence and microwave spectroscopy," Journal of Optical Technology 85 (2) 63-72 (2018).

[16] S. Osswald, G. Yushin, V. Mochalin, S. O. Kucheyev, and Y. Gogotsi, "Control of $\mathrm{sp} 2 / \mathrm{sp} 3$ carbon ratio and surface chemistry of nanodiamond powders by selective oxidation in air,” J. Am. Chem. Soc. 128(35), 11635-11642 (2006).

[17] A. I. Shames, V. Yu. Osipov, K. V. Bogdanov, A. V. Baranov, M. V. Zhukovskaya, A. Dalis, S. S. Vagarali, and A. Rampersaud, "Does progressive nitrogen doping intensify 
negatively charged nitrogen vacancy emission from e-beam-irradiated Ib type high-pressurehigh temperature diamonds?” J. Phys. Chem. C 121(9), 5232 (2017).

[18] J. F. Moulder, W. F. Stickle, P. E. Sobol, and K. D. Bomben, Handbook of X-ray Photoelectron Spectroscopy, J. Chastain, ed. (Perkin-Elmer Corp., Minnesota, 1992).

[19] Loubser J.H.N., van Wyk J.A., "Electron spin resonance in the study of diamond," Rep. Prog. Phys. 41, 1201-1248 (1978).

[20] A. I. Shames, V. Yu. Osipov, J. P. Boudou, A. M. Panich, H. J. von Bardeleben, F. Treussart, and A. Ya. Vul', "Magnetic resonance tracking of fluorescent nanodiamond fabrication,” J. Phys. D: Appl. Phys. 48(15), 155302 (2015).

[21] W. V. Smith, P. P. Sorokin, I. L. Gelles, and G. J. Lasher, "Electron spin resonance of nitrogen donors in diamond," Phys. Rev. 115(6), 1546 (1959).

[22] A. E. Aleksenskii, V. Yu. Osipov, A. Ya. Vul', B. Ya. Ber, A. B. Smirnov, V. G. Melekhin, G. J. Adriaenssens, and K. Iakoubovskii, "Optical properties of nanodiamond layers,” Phys. Solid State 43(1), 145-153 (2001) [Fiz. Tverd. Tela 43(1), 140-145 (2001)].

[23] V. Yu. Osipov, N. M. Romanov, F. M. Shakhov, and K. Takai, "Identifying quasi-free and bound nitrate ions on the surfaces of diamond nanoparticles by IR and x-ray photoelectron spectroscopy," Journal of Optical Technology 85 (3) 122-129 (2018).

[24] S. Osswald, V. N. Mochalin, M. Havel, G. Yushin, and Y. Gogotsi, "Phonon confinement effects in the Raman spectrum of nanodiamond," Phys. Rev. B 80, 075419 (2009).

[25] S. Stehlik, M. Varga, M. Ledinsky, D. Miliaieva, H. Kozak, V. Skakalova, C. Mangler, T. J. Pennycook, J. C. Meyer, A. Kromka and B. Rezek, "High-yield fabrication and properties of $1.4 \mathrm{~nm}$ nanodiamonds with narrow size distribution, "Sci. Rep. 6, 38419 (2016). 
[26] V. Mochalin, S. Osswald, and Y. Gogotsi, "Contribution of functional groups to the Raman spectrum of nanodiamond powders," Chem. Mater. 21 (2), 273-279 (2009).

[27] V.Yu. Osipov, A.M. Panich, A.V. Baranov, "Comment on "Carbon structure in nanodiamonds elucidated from Raman spectroscopy" by V.I. Korepanov et al.," Carbon 127, 193-194 (2018).

[28] R. J. Epstein, F. M. Mendoza, Y. K. Kato, and D. D. Awschalom, "Anisotropic interactions of a single spin and dark-spin spectroscopy in diamond," Nat. Phys. 1(2), 94-98 (2005).

[29] S. K. Sarkar, A. Bumb, X. Wu, K. a Sochacki, P. Kellman, M. W. Brechbiel, and K. C. Neuman, "Wide-field in vivo background free imaging by selective magnetic modulation of nanodiamond fluorescence.," Biomed. Opt. Express 5(4), 1190-1202 (2014).

[30] R. Chapman and T. Plakhoitnik, "Background-free imaging of luminescent nanodiamonds using external magnetic field for contrast enhancement," Optics Letters 38 ( 11), 1847-1849 (2013).

[31] J.-P. Tetienne, L. Rondin, P. Spinicelli, M. Chipaux, T. Debuisschert, J.-F. Roch and V. Jacques, "Magnetic-field-dependent photodynamics of single NV defects in diamond: an application to qualitative all-optical magnetic imaging," New Journal of Physics 14, 103033 (2012). 


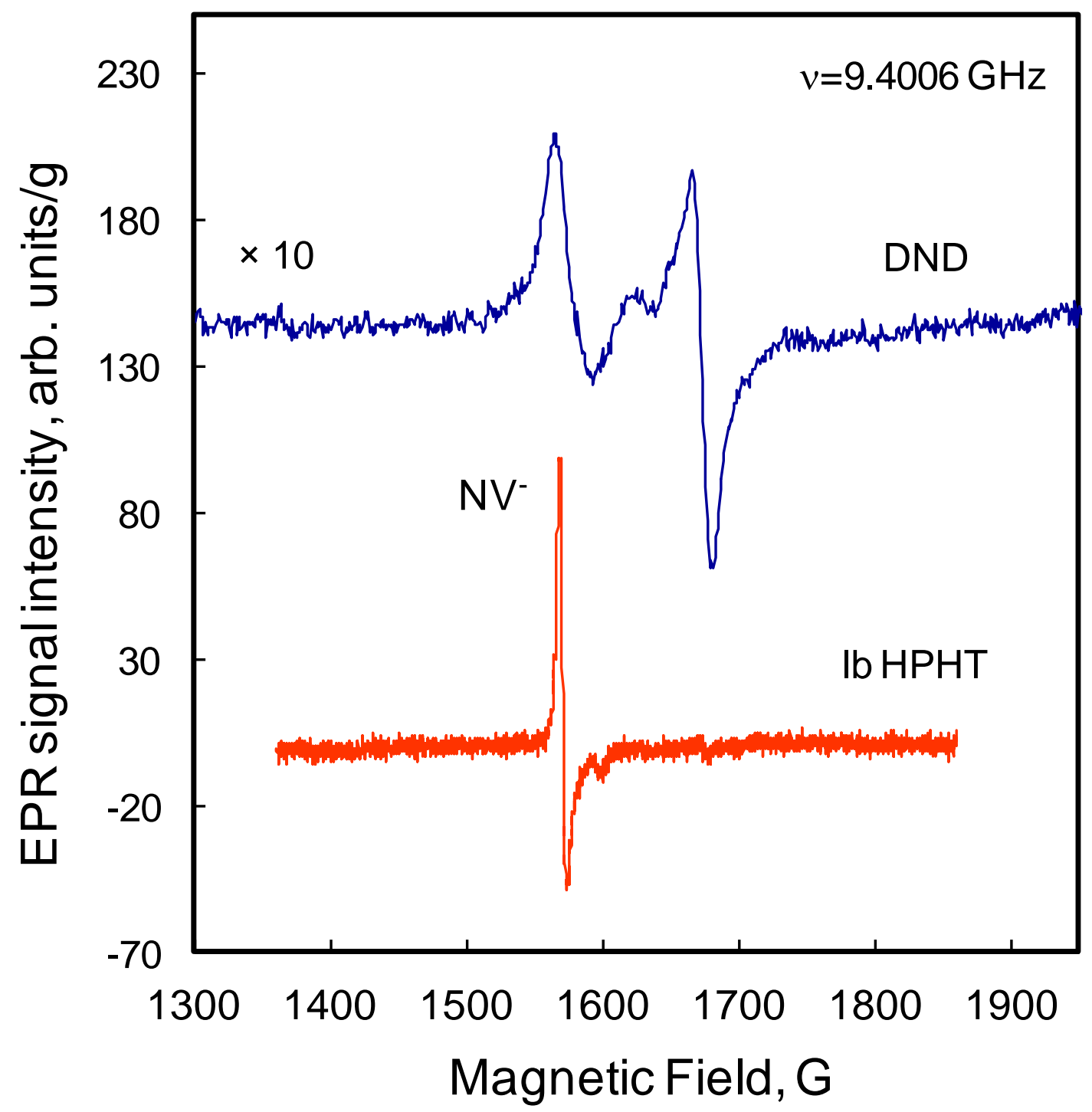

Figure 1 
(a)

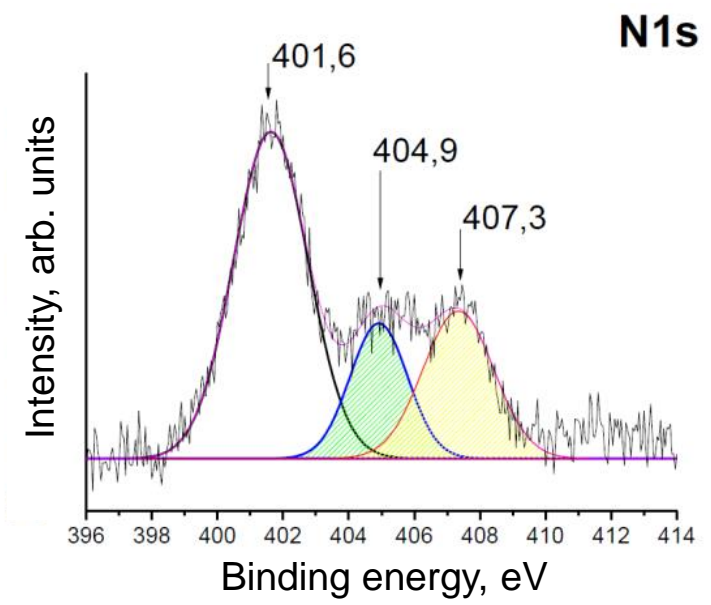

(b)

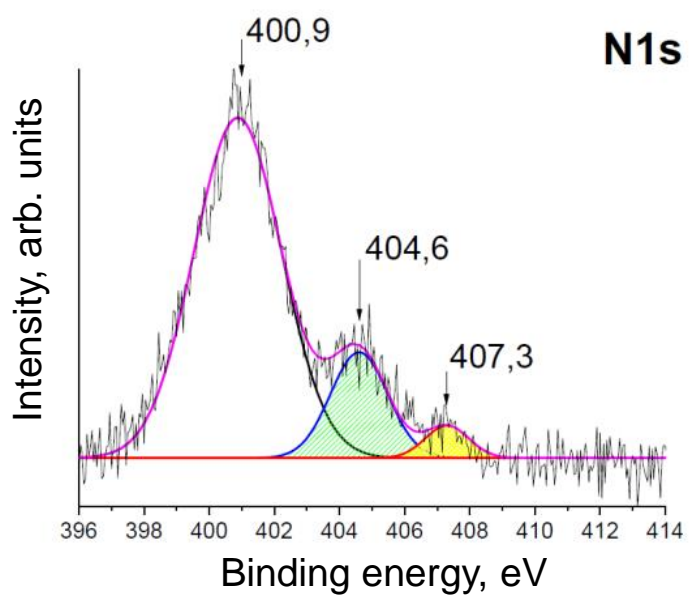

Figure 2 


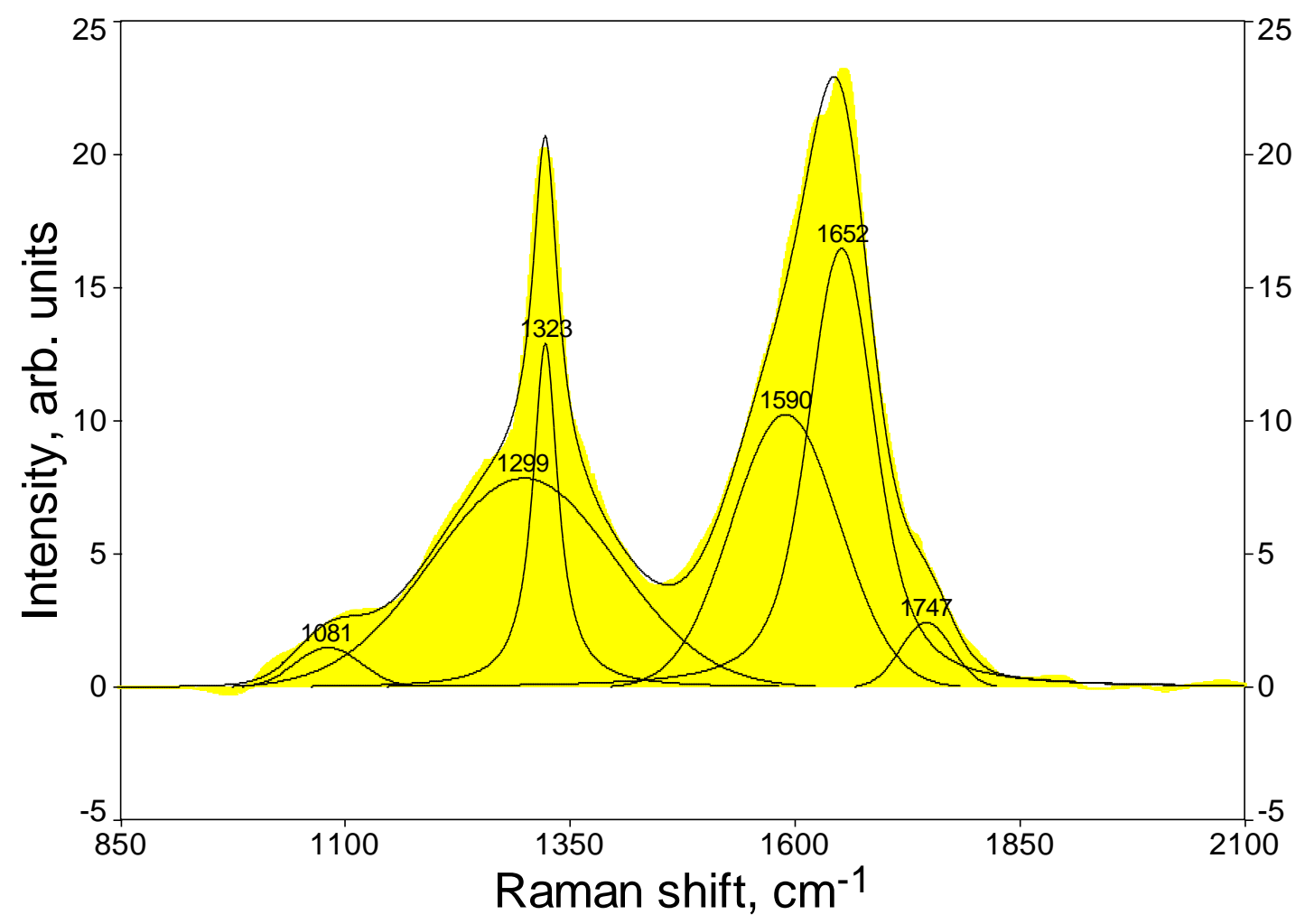

Figure 3 

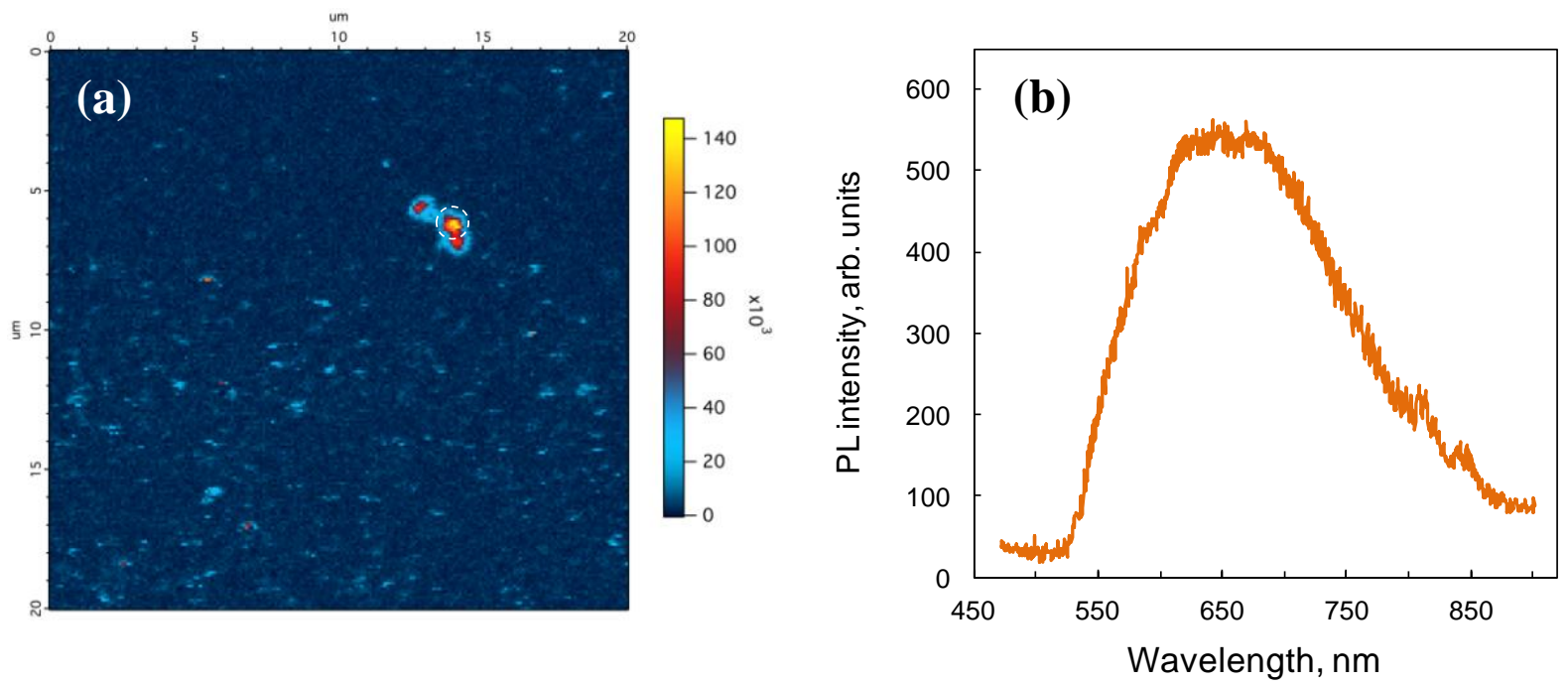

Figure 4 


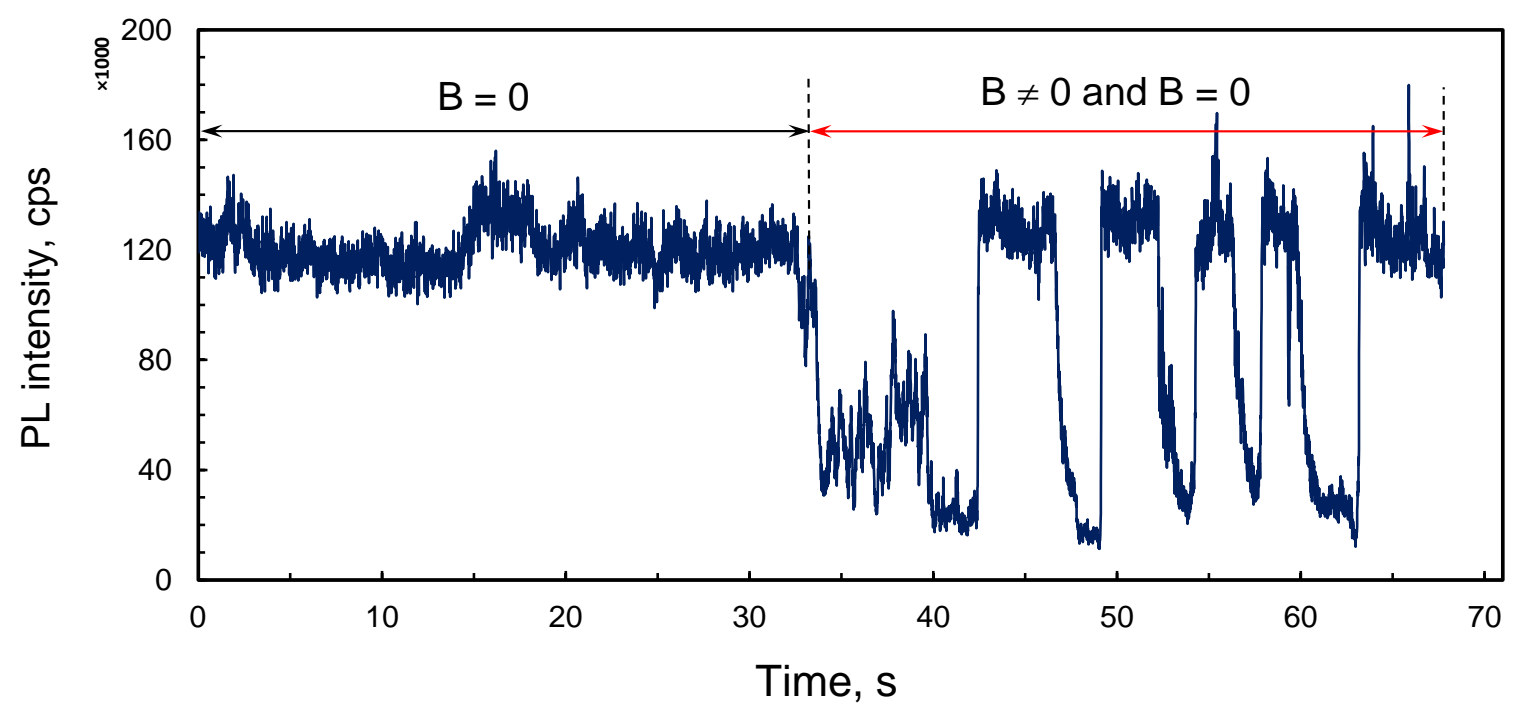

Figure 5 